\title{
Language lateralization with resting-state and task-based functional MRI in pediatric epilepsy
}

\author{
Virendra R. Desai, MD, ${ }^{1}$ Aditya Vedantam, MD, ${ }^{1}$ Sandi K. Lam, MD, MBA, ${ }^{1}$ Lucia Mirea, PhD, ${ }^{2}$ \\ Stephen T. Foldes, PhD, ${ }^{3}$ Daniel J. Curry, MD, ${ }^{1}$ P. David Adelson, MD, ${ }^{4,5}$ Angus A. Wilfong, MD, ${ }^{5}$ \\ and Varina L. Boerwinkle, MD ${ }^{5}$
}

\begin{abstract}
${ }^{1}$ Division of Pediatric Neurosurgery, Texas Children's Hospital/Department of Neurosurgery, Baylor College of Medicine, Houston, Texas; '2Department of Research, Phoenix Children's Hospital, Phoenix, Arizona; ${ }^{3}$ Department of Neuroscience Research, ${ }^{4}$ Division of Pediatric Neurosurgery, and ${ }^{5}$ Division of Pediatric Neurology, Barrow Neurological Institute at Phoenix Children's Hospital, Phoenix, Arizona
\end{abstract}

\begin{abstract}
OBJECTIVE Determining language laterality in patients with intractable epilepsy is important in operative planning. Wada testing is the gold standard, but it has a risk of stroke. Both Wada and task-based functional MRI (tb-fMRI) require patient cooperation. Recently, resting-state fMRI (rs-fMRI) has been explored for language lateralization. In the present study, the correlation between rs-fMRI and tb-fMRI in language lateralization is estimated in a pediatric population with intractable epilepsy.
\end{abstract}

METHODS rs-fMRI and tb-fMRI language lateralization testing performed as part of epilepsy surgery evaluation was retrospectively reviewed.

RESULTS Twenty-nine patients underwent rs-fMRI and tb-fMRI; a total of 38 rs-fMRI studies and 30 tb-fMRI studies were obtained. tb-fMRI suggested left dominance in 25 of 30 cases (83\%), right in $3(10 \%)$, and in $2(7 \%)$ the studies were nondiagnostic. In rs-fMRI, 26 of 38 studies (68\%) suggested left dominance, $3(8 \%)$ right dominance, $6(16 \%)$ bilateral, and $3(8 \%)$ were nondiagnostic. When tb-fMRI lateralized to the left hemisphere (25 cases), rs-fMRI was lateralized to the left in 23 patients $(92 \%)$ and it was bilateral/equal in $2(8 \%)$. When tb-fMRI lateralized to the right ( 3 cases), rs-fMRI lateralized to the right in all cases (100\%). The overall concordance rate was $0.93(95 \% \mathrm{Cl} 0.76-0.99)$ when considering cases with tb-fMRI and rs-fMRI performed within 6 months of each other, and tb-fMRI results were not nondiagnostic.

CONCLUSIONS rs-fMRI significantly correlated with tb-fMRI in lateralizing language and suggests the potential role for identifying hemispheric dominance via rs-fMRI. Further investigation and validation studies are warranted.

https://thejns.org/doi/abs/10.3171/2018.7.PEDS18162

KEYWORDS resting-state functional MRI; resting-state fMRI; task-based functional MRI; task-based fMRI; language lateralization; pediatric epilepsy; surgical technique

$\mathrm{T}$ HE incidence of pediatric epilepsy ranges from $23-$ 102 per 100,000 individuals in the US, with up to one-third of these cases remaining medically refractory ${ }^{14,22}$ In these cases, various surgical techniques may be utilized to achieve seizure freedom, including resection, functional disconnection, stereotactic laser ablation, vagal nerve stimulation, and reciprocal neurostimulation. ${ }^{10,18,29}$ Especially given the increased frequency of atypical language organization in this patient population, determining language laterality and localization in pediatric epilepsy cases is important in surgical evaluation and operative planning to optimize outcome and limit potential morbid- ity. ${ }^{2,25,28}$ Preservation of language abilities significantly improves postoperative quality of life. ${ }^{8}$

While the gold standard for language lateralization preoperatively has traditionally been Wada testing, its invasiveness, risk of stroke, and requirement of patient cooperation limit its use in pediatric patients. ${ }^{8}$ Task-based functional MRI (tb-fMRI) has emerged as an alternative, with favorable results compared to intraoperative cortical mapping in adults and relative to Wada testing. ${ }^{11,20,27}$ However, in the presence of brain tumors and extratemporal epileptic foci, correlation between tb-fMRI and direct cortical stimulation is less reliable and may even be

ABBREVIATIONS BOLD = blood oxygen level-dependent; fMRI = functional MRI; rs-fMRI = resting-state fMRI; RSN = resting-state network; tb-fMRI = task-based fMRI. SUBMITTED April 16, 2018. ACCEPTED July 24, 2018.

INCLUDE WHEN CITING Published online October 19, 2018; DOI: 10.3171/2018.7.PEDS18162. 
conflicting at times, highlighting the need for additional functional studies in the pediatric patient population..$^{13,26}$

In addition, tb-fMRI has limited use in patients with language barriers secondary to young age, cognitive disability, or altered consciousness such as that from anesthesia or coma. ${ }^{1,7,17,24}$ Although some studies have shown the potential for fMRI to lateralize language in infants through an auditory task that involves listening to speech, the reliability of this technique has not been validated against other measures. This difficulty in validation is understandable given an infant's inability to participate in other task-based and cooperation-dependent approaches such as Wada testing. ${ }^{6,24}$

Recently, resting-state fMRI (rs-fMRI) has been employed to lateralize language in healthy adults, healthy children, and adult patients with temporal lobe epilepsy and medically intractable focal epilepsy. ${ }^{7,8,28}$ To our knowledge, a similar undertaking in children with epilepsy is lacking. rs-fMRI utilizes mathematical techniques to define intrinsic activity in functionally connected language networks via small fluctuations in blood oxygen level-dependent (BOLD) signal throughout the brain.7.19 Brain regions showing synchronized oscillations in the low-frequency range $(<0.1 \mathrm{~Hz})$ are thought to be functionally connected as a resting-state network (RSN). ${ }^{17}$ This technique has been shown both to differentiate areas of initial seizure propagation from further downstream epileptogenic foci in children with known ictal onset from hypothalamic hamartoma ${ }^{4}$ and to localize epileptogenic zones that correlate with seizure freedom in a large pediatric epilepsy population. ${ }^{3}$ In this study, we sought to determine the correlation between rs-fMRI and tb-fMRI in language lateralization in a pediatric population with intractable epilepsy.

\section{Methods}

\section{Patient Population}

All pediatric epilepsy cases in which rs-fMRI was performed at our institution between April 11, 2012, and August 10, 2016, were retrospectively analyzed. Approval was obtained by the local institutional review board, with the determination that since the rs-fMRI studies were collected as part of a standard preoperative clinical MRI workup, no additional consent procedures were necessary due to minimal risk. Although specific consent for the rsfMRI sequence was not obtained, all patient families were made aware that their children were undergoing rs-fMRI. The de-identified clinical reports for tb-fMRI and rsfMRI were reviewed for those who met the initial exclusion criteria. These exclusion criteria were the following: sedation during fMRI as these patients were less likely to have tb-fMRI performed and age less than 10 years at the time of rs-fMRI as these patients were also likely to have been sedated. Children under the age of 10 years were also excluded because most of these patients had poor ability to cooperate with the required fMRI tasks, making the results of their tb-fMRI unreliable. Overall, 61 patients met the inclusion criteria of nonsedated rs-fMRI over the age of 10 during the study time period. Chart reviews were then conducted on these patients to determine if tb-fMRI was also performed, yielding 29 patients who had both rsfMRI and tb-fMRI studies performed in close temporal proximity.

\section{Data Acquisition}

Images were acquired on a 3-T MRI unit (Ingenuity, Philips Medical Systems) equipped with a 32-channel head coil. tb-fMRI parameters included TR $2000 \mathrm{msec}$, TE $30 \mathrm{msec}$, matrix size $64 \times 64$, flip angle 73, number of slices 32 , slice thickness $3.75 \mathrm{~mm}$ with 1-mm gap, 3.75 $\times 3.75-\mathrm{mm}$ in-plane resolution, and field of view $24 \times 24$ $\times 15.1 \mathrm{~cm}$. rs-fMRI parameters included TR $2000 \mathrm{msec}$, TE $30 \mathrm{msec}$, matrix size $80 \times 80$, flip angle 80 , number of slices 46 , slice thickness $3.4 \mathrm{~mm}$ with no gap, in-plane resolution $3 \times 3 \mathrm{~mm}$, and number of total volumes 600 lasting 20 minutes, well exceeding the accepted minimum required for analysis. ${ }^{5}$ For anatomical reference, a T1-weighted turbo field echo whole-brain sequence was obtained with the following parameters: TR $9 \mathrm{msec}$, TE $4 \mathrm{msec}$, flip angle 8 , slice thickness $0.9 \mathrm{~mm}$, and in-plane resolution $0.9 \times 0.9 \mathrm{~mm}$.

\section{Experimental Paradigm}

Awake tb-fMRI language testing occurred over several 5-minute sequences using the SensaVue system (Invivo). All patients underwent a finger-tapping task to establish the reliability of the exam and language tasks. Standard language paradigms included word listening, verb generation, word generation, and sentence completion. Only English speakers were included in this study as there is currently no standardized protocol in other languages. Details of each task are detailed in Table 1.

For rs-fMRI, patients were not given specific instructions other than to be still and have their eyes closed.

\section{rs-fMRI Processing}

Image analysis performed by Oxford's FSL (FMRIB Software Library) Model-Free FMRI Analysis (multivariate exploratory linear optimized decomposition into independent components, or MELODIC), probabilistic independent component analysis, and standard preprocessing steps were applied: The first 5 volumes were deleted to allow T1 signal saturation, high pass filter $100 \mathrm{sec}$, interleaved slice time correction, spatial smoothing of 1 $\mathrm{mm}$, and motion corrected by MCFLIRT, ${ }^{16}$ with nonbrain structures removed. Maximal head displacement varied between cases from 0.25 to $1 \mathrm{~mm}$.

\section{tb-fMRI Processing}

Images were processed using DynaSuite Neuro Software (Invivo Corporation), which involves motion correction, registration of the fMRI data set with T1-weighted MR images, and generalized linear model processing of block paradigm type designs.

\section{Language Lateralization}

In tb-fMRI, language laterality was defined for each patient as left significantly greater than right, right significantly greater than left, not obvious (i.e., bilateral/equal), or 
TABLE 1. Summary of tb-fMRI language tasks

\begin{tabular}{|c|c|c|}
\hline Task & Details & Duration (mins) \\
\hline Finger tapping & One hand performs at a time w/ alternating periods of tapping \& resting w/ eyes open \& the rest of the body still. & 4 \\
\hline Word listening & $\begin{array}{l}\text { A "+" sign appears on the screen followed by transient scanner noise, then by a series of words. The patient is } \\
\text { instructed to listen to the words \& try to understand them while resting w/ eyes open. }\end{array}$ & 4 \\
\hline Verb generation & $\begin{array}{l}\text { A "\#\#\#\#" symbol is presented on screen initially, \& the patient is instructed to not think of any word or sentence. } \\
\text { Then, a noun is presented on screen, \& the patient is required to think of a verb associated w/ it w/o saying } \\
\text { this verb or moving his/her lips. Examples are given prior to the presentation of the nouns. }\end{array}$ & 4 \\
\hline Word generation & $\begin{array}{l}\text { A letter is presented the screen, \& patient is required to think of a word beginning w/ that letter w/o saying this } \\
\text { word or moving his/her lips. }\end{array}$ & 4 \\
\hline $\begin{array}{l}\text { Sentence gen- } \\
\text { eration }\end{array}$ & $\begin{array}{l}\text { Initially, a series of nonsense letters is presented, \& the patient is instructed to rest w/ eyes open. Then an Eng- } \\
\text { lish sentence w/ a blank for one word is presented, \& the patient is required to read the sentence to himself/ } \\
\text { herself \& think of a word that fills in the blank w/o speaking out loud or moving his/her lips. Examples are } \\
\text { given prior to the presentation for the fill-in-the-blank sentences. }\end{array}$ & 4 \\
\hline
\end{tabular}

nondiagnostic, as seen in Fig. 1. A board-certified pediatric neuroradiologist assessed functional parametric maps at multiple thresholds with no specific $\mathrm{p}$ value enforced. Dominance was visually determined by comparing activation in inferior and middle frontal gyri as well as the inferior parietal lobule with activation on the opposite side. In rs-fMRI, laterality was also categorized as above and analyzed by the senior author (V.L.B.), a board-certified pediatric neurologist with dedicated expertise in rs-fMRI, with 7 years of experience, as seen in Fig. 2.

The results of rs-fMRI independent component analysis were first sorted according to the criteria previously described. ${ }^{3}$ RSNs, which included areas of the inferior frontal gyrus, supramarginal gyrus, superior temporal gyrus, and the superior temporal sulcus in either hemisphere were overlapped onto the patient's anatomical image. Lateralization was determined by ranked criteria, as described previously in Boerwinkle et al. in 2017, ${ }^{3}$ including primarily the greatest visual spatial extent of the described areas, long-range receptive and expressive language area within a single RSN, and contextual grading of the RSN signal source within all the patient's signal sources. If a signal source was itself significantly overlapping one of these major language areas but was determined to be an epileptogenic zone signal source, according to our previously published criteria, ${ }^{3}$ then this signal source was excluded from the determination of language lateralization, as seen in Fig. 2.

Data for several demographic and baseline clinical variables were collected by chart review. Associations between rs-fMRI and tb-fMRI laterality diagnosis were examined using the Fisher exact test. In addition, the concordance rate in laterality diagnosis between rs-fMRI and tb-fMRI was estimated, and 95\% confidence intervals were computed to indicate uncertainty in the data.

\section{Results}

During the study period, a total of 38 rs-fMRI studies and 30 tb-fMRI studies were performed. Both rs-fMRI and tb-fMRI were performed in 29 patients. Demographic data are shown in Table 2, with seizure diagnosis based on etiological classification as reported by Shorvon et al. ${ }^{23}$ Findings of rs-fMRI and tb-fMRI are presented in Table
3. Three rs-fMRI studies were nondiagnostic and required repeating, while two tb-fMRIs were nondiagnostic and required repeating. Some patients had multiple studies performed, which were all included even if the diagnosis was the same in both. Thus, in 29 total patients, 38 rs-fMRI studies and 30 tb-fMRI studies were performed.

tb-fMRI suggested left dominance in 25 of 30 cases $(83 \%)$, right dominance in $3(10 \%)$, and findings were nondiagnostic in $2(7 \%)$. No patient had bilateral dominance on tb-fMRI. rs-fMRI suggested left dominance in 26 of 38 cases $(68 \%)$, right dominance in 3 cases $(8 \%)$, and results were nondiagnostic in 3 cases (8\%); interestingly, 6 cases
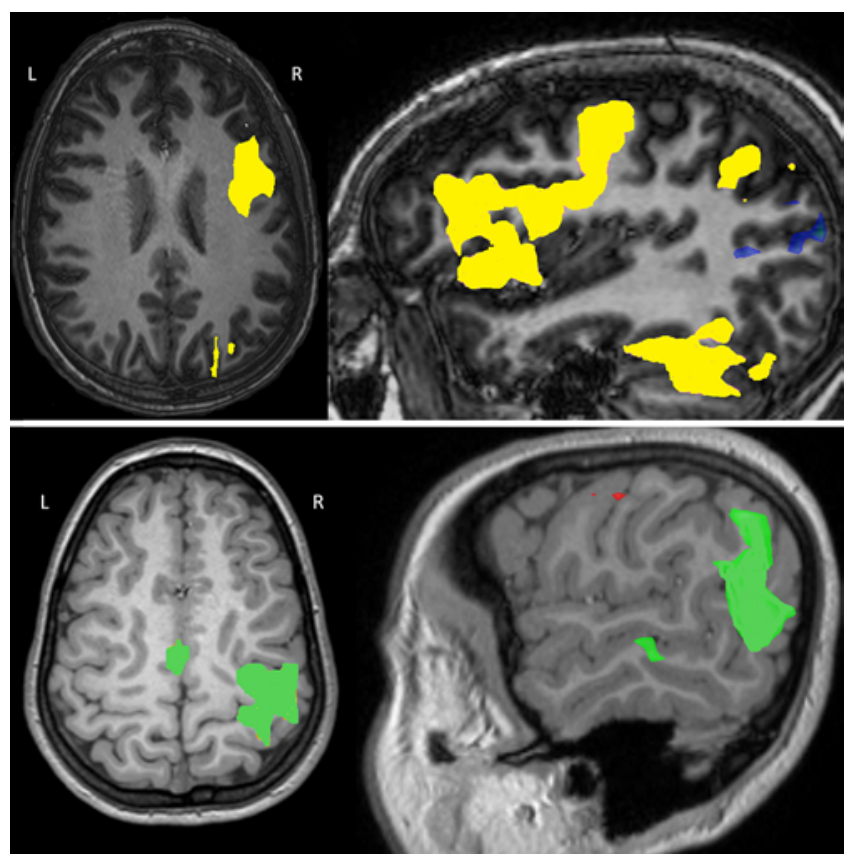

FIG. 1. An example of task-based language lateralization. Upper: Axial and sagittal images depicting expressive language function during tasks such as verb and word generation and sentence completion. Lower: Axial and sagittal images demonstrating receptive language function during tasks such as word and story listening. Studies demonstrated right-hemispheric dominance in this patient. Figure is available in color online only. 


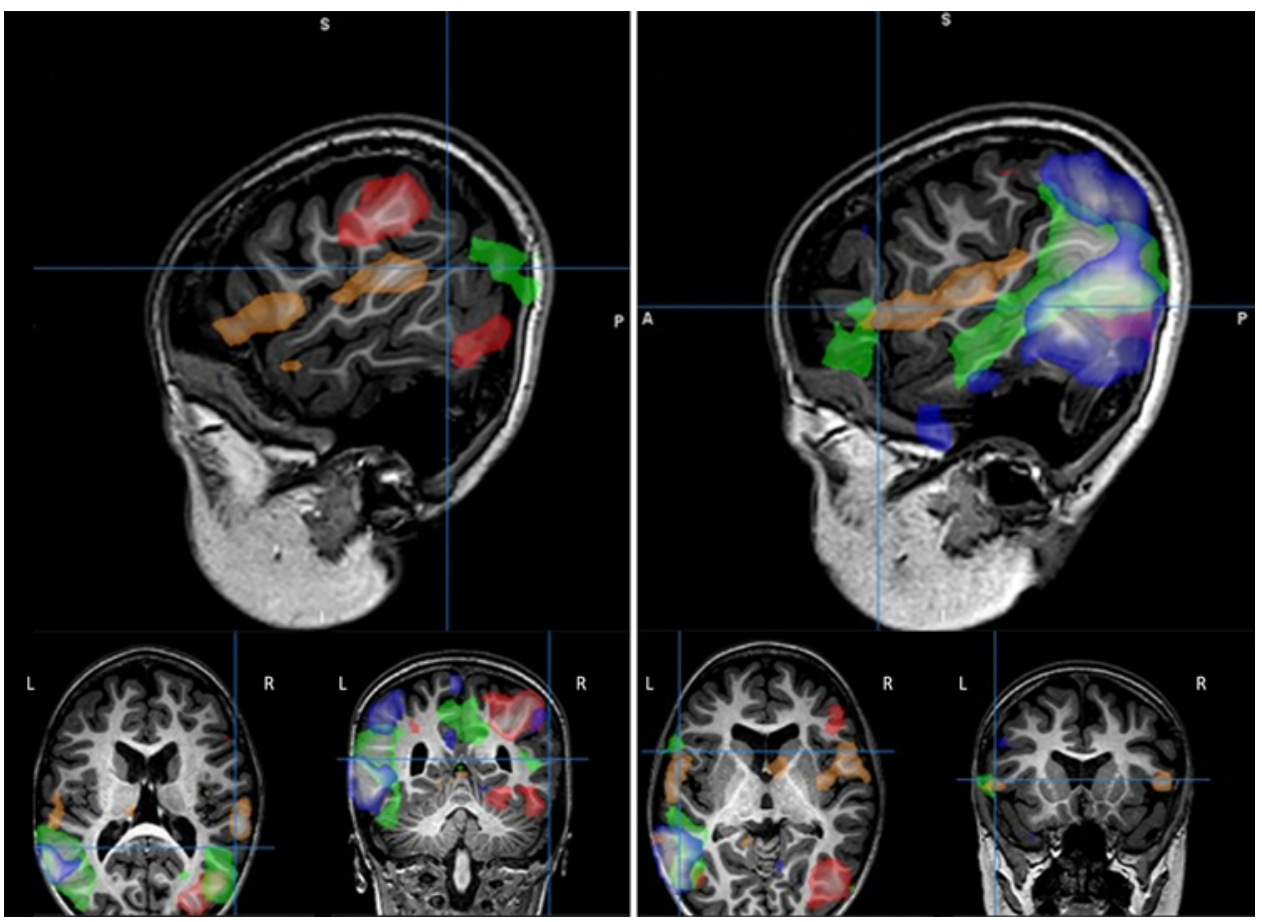

FIG. 2. An example of resting-state language lateralization. Left and right: Axial, coronal, and sagittal images showing the patient's language-area RSNs overlapping, each in a different color. The images on the right in the two panels are windowed to show the same patient's left sagittal language view of the RSNs. The language network areas are spatially clearly far more extensive in the left rather than the right hemisphere, and this patient was determined to have left-hemispheric language dominance. Figure is available in color online only.

(16\%) were found to be bilateral. In 8 patients, rs-fMRI was performed but tb-fMRI was not performed in close enough temporal proximity. Thus, there were 30 total cases in which both rs-fMRI and tb-fMRI were performed in close temporal proximity.

There was a significant association between rs-fMRI and tb-fMRI lateralization diagnoses $(\mathrm{p}<0.0001$, Fisher

TABLE 2. Summary demographic and seizure data for 29 patients

\begin{tabular}{lc}
\hline \multicolumn{1}{c}{ Characteristic } & Value \\
\hline Median age (IQR), yrs & $16.3(13.2-17.0)$ \\
\hline Sex ratio (M/F), no. & $12: 17$ \\
\hline Handedness ratio (left/right), no. & $6: 23$ \\
\hline Seizure diagnosis, no. (\%) & \\
\hline Symptomatic localization-related epilepsy & $20(69 \%)$ \\
\hline Idiopathic & $5(17 \%)$ \\
\hline Cryptogenic localization-related epilepsy & $4(14 \%)$ \\
\hline Provoked & $0(0 \%)$ \\
\hline No. of antiepileptic drugs (\%) & $8(28 \%)$ \\
\hline 1 & $13(45 \%)$ \\
\hline 2 & $5(17 \%)$ \\
\hline 3 & $1(3 \%)$ \\
\hline 4 & $2(7 \%)$ \\
\hline Unknown
\end{tabular}

${ }^{*}$ Based on etiological classification. ${ }^{23}$ exact test). Table 4 presents the concordance of the rsfMRI and tb-fMRI results obtained in the 29 patients. When tb-fMRI lateralized to the left (25 cases), rs-fMRI also lateralized to the left in $23(92 \%)$ and was bilateral/ equal in $2(8 \%)$. When tb-fMRI lateralized to the right (3 cases), rs-fMRI lateralized to the right in all cases (100\%). When tb-fMRI was nondiagnostic ( 2 cases), rs-fMRI lateralized to the left in $1(50 \%)$ and was bilateral/equal in 1 $(50 \%)$.

After excluding the 2 cases in which tb-fMRI was nondiagnostic, rs-fMRI indicated the same laterality as tb-fMRI in 26 of 28 cases, resulting in an estimated concordance rate of 0.93 (95\% CI $0.78-0.98$ ). We noted that the 2 of 28 cases were not discordant for lateralization but, rather, were classified as bilateral/equal by the rs-fMRI (Table 5).

TABLE 3. Overall results of rs-fMRI and tb-fMRI studies

\begin{tabular}{lrrrrr}
\hline & \multicolumn{3}{c}{ Study } \\
\cline { 2 - 3 } \cline { 5 - 6 } \cline { 5 - 6 } \cline { 5 - 6 } Laterality & \multicolumn{2}{c}{ No.fMRI } & & \multicolumn{2}{c}{ tb-fMRI } \\
\cline { 2 - 3 } \cline { 5 - 6 } Left & 26 & $68 \%$ & & 25 & $83 \%$ \\
\hline Right & 3 & $8 \%$ & & 3 & $10 \%$ \\
\hline Bilateral/equal & 6 & $16 \%$ & & 0 & $0 \%$ \\
\hline Nondiagnostic & 3 & $8 \%$ & & 2 & $7 \%$ \\
\hline Total & 38 & & & 30 & \\
\hline
\end{tabular}


TABLE 4. Concordance of rs-fMRI and tb-fMRI results

\begin{tabular}{|c|c|c|c|c|c|c|}
\hline \multirow[b]{2}{*}{ rs-fMRI } & \multicolumn{5}{|c|}{ tb-fMRI, No. (\%) } & \multirow[b]{2}{*}{ Other' } \\
\hline & Right & Left & Bilateral/Equal & Nondiagnostic & Total & \\
\hline Right & $3(100 \%)$ & 0 & 0 & 0 & 3 & 0 \\
\hline Left & 0 & $23(92 \%)$ & 0 & $1(50 \%)$ & 24 & 2 \\
\hline Bilateral/equal & 0 & $2(8 \%)$ & 0 & $1(50 \%)$ & 3 & 3 \\
\hline Nondiagnostic & 0 & 0 & 0 & 0 & 0 & 2 \\
\hline Total & 3 & 25 & 0 & 2 & 30 & \\
\hline Other* & 0 & 0 & 0 & 0 & & \\
\hline
\end{tabular}

* Other category represents instances when rs-fMRI was performed at a time point during which no tb-fMRI study was acquired in close temporal proximity (or vice versa). If a patient had two rs-fMRI studies, but one tb-fMRI study, the rs-fMRI study obtained closest in time to the tb-fMRI study was categorized according to tb-fMRI lateralization, while the other rs-fMRI scan was placed in the "other" category.

In this study, $22(76 \%)$ patients had 1 rs-fMRI scan, $5(17 \%)$ had 2 , and $2(7 \%)$ had 3 , as deemed necessary by their clinician according to ongoing clinical changes in the patient. In contrast, 28 (97\%) of the 29 patients had a single tb-fMRI scan, and 1 patient (3\%) had 2.

On standard anatomical MRI, 6 patients (21\%) had mesial temporal sclerosis only, 1 (3\%) had mesial temporal sclerosis along with a malacic cavity secondary to a chronic insult, $1(3 \%)$ had malacic cavity due to a chronic insult only, 8 (28\%) had focal cortical dysplasia, $6(21 \%)$ had tumors, $1(3 \%)$ had a cavernoma, 4 (14\%) had normal findings, and $2(7 \%)$ had stigmata of tuberous sclerosis (tubers and subependymal nodules).

\section{Discussion}

In this study, we found an overall concordance rate of 0.93 (95\% CI 0.76-0.99) between tb-fMRI and rs-fMRI. Importantly, when tb-fMRI lateralized to a certain hemisphere, rs-fMRI did not lateralize to the contralateral hemisphere in any case. For instance, there were 2 cases (8\%) in which tb-fMRI lateralized to the left hemisphere and rsfMRI suggested bilateral or equal dominance, rather than incorrectly lateralizing to the contralateral hemisphere. Incorrect lateralization could potentially give the surgeon a false sense of security when operating near the speech and language area. In our study, 13 of the patients went on to undergo resection, 4 to have a vagal nerve stimulator placed, 5 to have ablation, and 7 to undergo no surgery. In all cases, language lateralization guided surgical management, and none of the patients suffered long-term, surgically induced speech deficit. Neither of the 2 patients with differing fMRI results underwent any surgery.

Intractable epilepsy has numerous long-term consequences that multiply the longer it remains untreated. ${ }^{12}$ Therefore, surgical intervention is warranted as early as possible in medically refractory cases. In some instances, this requires operating on very young children, who have not yet cognitively developed language function advanced enough to participate in tb-fMRI, although they possess the ability to do so given no interference from epilepsy or other pathology. Language centers reorganize during development and can also be affected by epilepsy, especially at younger ages. ${ }^{15,25}$ In these cases, rs-fMRI may represent a powerful method of determining language laterality and providing vital preoperative information to the surgeon. Notably, as mentioned above, ongoing clinical changes were more frequently assessed by rs-fMRI than tb-fMRI, given our belief in the reliability of the former at our institution.

A previous study analyzed correlations between rsfMRI and Wada testing in young adult patients (mean age of $27.2 \pm 13.1$ years [range 10-59 years]) and found a $96 \%$ accuracy rate and $96 \%$ sensitivity and specificity? In the current study, we examined the agreement between rsfMRI and tb-fMRI to detect language lateralization in the pediatric epilepsy setting in patients older than 10 years of age. Younger patients were not studied but remain an opportunity to better identify language development in this unique population, with numerous cortical and other brain anomalies contributing to their seizure syndrome.

With a 93\% concordance for rs-fMRI and tb-fMRI to detect language lateralization, the consistency of the rsfMRI results was high and even remained so when patients underwent repeat scanning. Of those patients who had multiple rs-fMRI studies performed, two had repeat scans because their initial scans were nondiagnostic. Of the remaining 6 such patients, 4 had consistent laterality between exams, while 2 had inconsistent results. However, the inconsistent results never led to the opposite hemisphere being diagnosed as the dominant one; instead, one scan suggested bilateral dominance and another suggested left-sided dominance. Notably, there was one patient who had an rs-fMRI scan in 2013 that suggested left-sided dominance and correlated with tb-fMRI results. In a repeat study 2 years later, the rs-fMRI scans suggested equal dominance. This stands in contrast to the typical natural history in which functional asymmetry, rather than symmetry, develops over time, especially from the end of the 1 st year through the 2 nd year of life.?

This present study has some limitations. While patients in this study could be studied with tb-fMRI, the ability of rs-fMRI to accurately predict hemispheric dominance in pediatric epilepsy patients is largely unexplored. Longitudinal analysis may help better determine its accuracy in this setting of older children, although language reorganization does occur over time during development, ${ }^{9,15}$ and therefore, younger patients need to be included in future studies to better understand language network development. In addition, while most rs-fMRI studies were 
TABLE 5. Summary of patients with repeat studies

\begin{tabular}{|c|c|c|c|}
\hline \multirow{2}{*}{$\begin{array}{l}\text { Case No.l } \\
\text { Study }\end{array}$} & \multicolumn{3}{|c|}{ Study No. } \\
\hline & First & Second & Third \\
\hline \multicolumn{4}{|l|}{1} \\
\hline rs-fMRI & $\begin{array}{l}\text { 6/12/2014: nearly equal, } \\
\text { slightly left }\end{array}$ & 7/12/2014: equal & \\
\hline tb-fMRI & $\begin{array}{l}\text { 6/27/2014: left }>\text { right } \\
\text { but fairly extensive } \\
\text { bilaterally }\end{array}$ & & \\
\hline \multicolumn{4}{|l|}{2} \\
\hline rs-fMRI & $\begin{array}{l}\text { 6/26/2013: left dominant } \\
\text { but nearly equal }\end{array}$ & $\begin{array}{c}\text { 1/1/2015: left } \\
\text { dominant }\end{array}$ & \\
\hline tb-fMRI & $\begin{array}{l}\text { 6/26/2013: auditory } \\
\text { listening only (equal); } \\
\text { nondiagnostic in } \\
\text { story listening }\end{array}$ & & \\
\hline \multicolumn{4}{|l|}{3} \\
\hline rs-fMRI & 6/24/2013: left & 1/6/2015: equal & $\begin{array}{l}\text { 10/8/2015: } \\
\text { equal }\end{array}$ \\
\hline tb-fMRI & 6/24/2013: left & & \\
\hline \multicolumn{4}{|l|}{4} \\
\hline rs-fMRI & $\begin{array}{l}\text { 1/30/2013: mostly left, } \\
\text { one network right }\end{array}$ & 3/1/2016: left & \\
\hline tb-fMRI & 1/14/2013: left & 10/21/2015: left & \\
\hline \multicolumn{4}{|l|}{5} \\
\hline rs-fMRI & $\begin{array}{l}\text { 6/12/2015: unreliable } \\
\text { due to oral cavity } \\
\text { artifact }\end{array}$ & $\begin{array}{l}\text { 5/19/2016: nearly } \\
\text { equal; right } \\
\text { dominant }\end{array}$ & \\
\hline tb-fMRI & $\begin{array}{l}\text { 6/10/2015: nondiagnos- } \\
\text { tic due to oral cavity } \\
\text { artifact }\end{array}$ & 5/11/2016: right & \\
\hline \multicolumn{4}{|l|}{6} \\
\hline rs-fMRI & $\begin{array}{l}\text { 1/9/2013: equal but } \\
\text { unreliable }\end{array}$ & 10/6/2014: left & $\begin{array}{l}\text { 4/27/2015: } \\
\quad \text { nondi- } \\
\text { agnostic } \\
\text { (too short) }\end{array}$ \\
\hline tb-fMRI & 10/6/2014: left & & \\
\hline \multicolumn{4}{|l|}{7} \\
\hline rs-fMRI & $\begin{array}{l}\text { 3/30/2015: left dominant } \\
\text { but receptive areas } \\
\text { greater on right }\end{array}$ & $\begin{array}{c}\text { 6/8/2016: left } \\
\text { dominant }\end{array}$ & \\
\hline tb-fMRI & 3/30/2015: left dominant & & \\
\hline
\end{tabular}

acquired on the same day as the tb-fMRI studies, some were obtained several days to weeks apart. We worked under the assumption that a time span of weeks rather than months would minimize bias. Moreover, the results of the rs-MRI studies were compared to those of the tb-fMRI studies; despite the latter being regarded as a reliable predictor of language laterality, there remains potential for discordance between these results and those obtained by more robust measures. We selected patients only with nonsedated rs-fMRI exams; however, some epilepsy patients require sedation to remain still in an MRI scanner for a prolonged time. ${ }^{1}$ Propofol anesthesia may attenuate rs-fMRI BOLD signal in adults, but the full extent of its impact on language lateralization in the pediatric setting is unknown. ${ }^{1,17}$

Future studies are warranted to validate our results. As expected, different tasks during tb-fMRI led to activation of different brain regions, such as Broca's area versus Wernicke's, and can lateralize more or less strongly. These areas can even lateralize to opposite hemispheres. ${ }^{20,30}$ As a result, combining various language paradigms can increase the validity of language lateralization of tb-fMRI. ${ }^{8,11}$ At our institution, 3 tasks - verb generation, word generation, and sentence completion-are typically performed, increasing the robustness of our tb-fMRI results. Despite this, our rs-fMRI results may benefit from more validation studies against more-robust methods such as Wada testing or direct cortical stimulation.

Future directions include analysis of rs-fMRI studies with patients sedated, with the aim of better delineating the effect of sedation on lateralization in pediatric epilepsy patients. Different brain regions can be used in rs-fMRI for language lateralization, such as Broca's and Wernicke's areas. ${ }^{8}$ While in our study, we employed activity within the inferior frontal gyrus, supramarginal gyrus, superior temporal gyrus, or the superior temporal sulcus to lateralize hemispheric dominance, future studies on the predictive capability of other brain regions should be performed. While lateralizing language is an important first step, the further localization of language function to specific cortical areas could further guide operative planning. Different pathologies can also affect language lateralization; for instance, lesions such as tumors, vascular malformations, and other congenital malformations can influence adjacent cerebral blood flow and thereby affect BOLD signal, leading to variable fMRI results. ${ }^{1,20}$ It is important that the accuracy of rs-fMRI in different pathologies be assessed, to understand its role in various pediatric epilepsy populations. Future steps may also include applying rs-fMRI for lateralizing memory function, as tb-fMRI has been used in an indirect manner for this purpose in children. ${ }^{21}$

\section{Conclusions}

Language lateralization with rs-fMRI was significantly associated with traditional tb-fMRI methods in our pediatric epilepsy population. The high concordance of 93\% suggests a role for rs-fMRI in identifying hemispheric dominance. Further investigation and validation studies are warranted to justify using rs-fMRI in conjunction with or in place of standard methods.

\section{References}

1. Altman NR, Bernal B: Pediatric applications of functional magnetic resonance imaging. Pediatr Radiol 45 (Suppl 3):S382-S396, 2015

2. Berl MM, Zimmaro LA, Khan OI, Dustin I, Ritzl E, Duke ES, et al: Characterization of atypical language activation patterns in focal epilepsy. Ann Neurol 75:33-42, 2014

3. Boerwinkle VL, Mohanty D, Foldes ST, Guffey D, Minard CG, Vedantam A, et al: Correlating resting-state functional magnetic resonance imaging connectivity by independent component analysis-based epileptogenic zones with intracranial electroencephalogram localized seizure onset zones and surgical outcomes in prospective pediatric intractable epilepsy study. Brain Connect 7:424-442, 2017 
4. Boerwinkle VL, Wilfong AA, Curry DJ: Resting-state functional connectivity by independent component analysis-based markers corresponds to areas of initial seizure propagation established by prior modalities from the hypothalamus.

Brain Connect 6:642-651, 2016

5. Damoiseaux JS, Rombouts SA, Barkhof F, Scheltens P, Stam CJ, Smith SM, et al: Consistent resting-state networks across healthy subjects. Proc Natl Acad Sci U S A 103:1384813853, 2006

6. Dehaene-Lambertz G, Dehaene S, Hertz-Pannier L: Functional neuroimaging of speech perception in infants. Science 298:2013-2015, 2002

7. DeSalvo MN, Tanaka N, Douw L, Leveroni CL, Buchbinder $\mathrm{BR}$, Greve DN, et al: Resting-state functional MR imaging for determining language laterality in intractable epilepsy. Radiology 281:264-269, 2016

8. Doucet GE, Pustina D, Skidmore C, Sharan A, Sperling MR, Tracy JI: Resting-state functional connectivity predicts the strength of hemispheric lateralization for language processing in temporal lobe epilepsy and normals. Hum Brain Mapp 36:288-303, 2015

9. Emerson RW, Gao W, Lin W: Longitudinal study of the emerging functional connectivity asymmetry of primary language regions during infancy. J Neurosci 36:10883-10892, 2016

10. Engel J Jr: Overview of surgical treatment for epilepsy, in Shorvon S, Perucca E, Engel J Jr (eds): The Treatment of Epilepsy, ed 4. New York: Wiley, 2015, pp 709-722

11. Gaillard WD, Balsamo L, Xu B, Grandin CB, Braniecki SH, Papero PH, et al: Language dominance in partial epilepsy patients identified with an fMRI reading task. Neurology 59:256-265, 2002

12. Gilliam F: A lighter shade of gray: is temporal lobe epilepsy a progressive gray matter disorder? Epilepsy Curr 17:23-24, 2017

13. Giussani C, Roux FE, Ojemann J, Sganzerla EP, Pirillo D, Papagno C: Is preoperative functional magnetic resonance imaging reliable for language areas mapping in brain tumor surgery? Review of language functional magnetic resonance imaging and direct cortical stimulation correlation studies. Neurosurgery 66:113-120, 2010

14. Hauser WA: Epidemiology of epilepsy in children, in Pellock JM, Nordli DR Jr, Sankar R, et al (eds): Pellock's Pediatric Epilepsy: Diagnosis and Therapy, ed 4. New York: Demos, 2016, pp 177-206

15. Holland SK, Vannest J, Mecoli M, Jacola LM, Tillema JM, Karunanayaka PR, et al: Functional MRI of language lateralization during development in children. Int J Audiol 46:533-551, 2007

16. Jenkinson M, Bannister P, Brady M, Smith S: Improved optimization for the robust and accurate linear registration and motion correction of brain images. Neuroimage 17:825-841, 2002

17. Kirsch M, Guldenmund P, Ali Bahri M, Demertzi A, Baquero K, Heine L, et al: Sedation of patients with disorders of consciousness during neuroimaging: effects on resting state functional brain connectivity. Anesth Analg 124:588-598, 2017

18. Lam S, Hadley C, Curry DJ, Pan IW: Comparison of perioperative costs between stereotactic laser ablation and craniotomy for hypothalamic hamartoma. J Pediatr Epilepsy 6:97-102, 2017

19. Liu H, Stufflebeam SM, Sepulcre J, Hedden T, Buckner RL: Evidence from intrinsic activity that asymmetry of the human brain is controlled by multiple factors. Proc Natl Acad Sci U S A 106:20499-20503, 2009

20. Ruff IM, Petrovich Brennan NM, Peck KK, Hou BL, Tabar $\mathrm{V}$, Brennan CW, et al: Assessment of the language laterality index in patients with brain tumor using functional MR imaging: effects of thresholding, task selection, and prior surgery. AJNR Am J Neuroradiol 29:528-535, 2008

21. Sepeta LN, Berl MM, Wilke M, You X, Mehta M, Xu B, et al: Age-dependent mesial temporal lobe lateralization in language fMRI. Epilepsia 57:122-130, 2016

22. Shinnar S, Pellock JM: Update on the epidemiology and prognosis of pediatric epilepsy. J Child Neurol 17 (Suppl 1):S4-S17, 2002

23. Shorvon SD: The etiologic classification of epilepsy. Epilepsia 52:1052-1057, 2011

24. Souweidane MM, Kim KH, McDowall R, Ruge MI, Lis E, Krol G, et al: Brain mapping in sedated infants and young children with passive-functional magnetic resonance imaging. Pediatr Neurosurg 30:86-92, 1999

25. Springer JA, Binder JR, Hammeke TA, Swanson SJ, Frost JA, Bellgowan PS, et al: Language dominance in neurologically normal and epilepsy subjects: a functional MRI study. Brain 122:2033-2046, 1999

26. Szaflarski JP, Gloss D, Binder JR, Gaillard WD, Golby AJ, Holland SK, et al: Practice guideline summary: Use of fMRI in the presurgical evaluation of patients with epilepsy: Report of the Guideline Development, Dissemination, and Implementation Subcommittee of the American Academy of Neurology. Neurology 88:395-402, 2017

27. Szaflarski JP, Holland SK, Jacola LM, Lindsell C, Privitera MD, Szaflarski M: Comprehensive presurgical functional MRI language evaluation in adult patients with epilepsy. Epilepsy Behav 12:74-83, 2008

28. Teghipco A, Hussain A, Tivarus ME: Disrupted functional connectivity affects resting state based language lateralization. Neuroimage Clin 12:910-927, 2016

29. Thomas GP, Jobst BC: Critical review of the responsive neurostimulator system for epilepsy. Med Devices (Auckl) 8:405-411, 2015

30. Wilke M, Pieper T, Lindner K, Dushe T, Holthausen H, Krägeloh-Mann I: Why one task is not enough: functional MRI for atypical language organization in two children. Eur J Paediatr Neurol 14:474-478, 2010

\section{Disclosures}

Dr. Curry reports being a consultant for Medtronic, Inc., and Monteris, Inc. Dr. Wilfong reports being a consultant for LivaNova. He receives support for non-study-related clinical or research efforts that he oversees from the following: LivaNova, UCB, Zogenix, and Greenwich Biosciences.

\section{Author Contributions}

Conception and design: Boerwinkle, Desai, Lam, Curry. Acquisition of data: Boerwinkle, Desai, Vedantam, Curry, Wilfong. Analysis and interpretation of data: Boerwinkle, Desai, Lam, Foldes, Adelson, Wilfong. Drafting the article: Desai. Critically revising the article: Boerwinkle, Desai, Vedantam, Lam, Foldes. Reviewed submitted version of manuscript: all authors. Approved the final version of the manuscript on behalf of all authors: Boerwinkle. Statistical analysis: Desai, Mirea. Study supervision: Boerwinkle, Lam.

\section{Supplemental Information \\ Previous Presentations}

A portion of this manuscript was presented at the American Association of Neurological Surgeons Annual Meeting, Los Angeles, CA, April 22-26, 2017.

\section{Correspondence}

Varina L. Boerwinkle: Phoenix Children's Hospital, Barrow Neurological Institute, Phoenix, AZ. vboerwinkle@ phoenixchildrens.com. 